\title{
Crucial function of histone lysine methylation in plant reproduction
}

\author{
YAO XiaoZhen ${ }^{1 *} \&$ SHEN WenHui ${ }^{2 *}$ \\ ${ }^{1}$ State Key Laboratory of Genetic Engineering, Department of Biochemistry, School of Life Sciences, Fudan University, Shanghai 200433, China; \\ ${ }^{2}$ Institut de Biologie Moléculaire des Plantes du CNRS, Université de Strasbourg, 12 rue du Général ZIMMER, 67084 STRASBOURG, France
}

Received June 15, 2011; accepted August 12, 2011

\begin{abstract}
Unlike animals, plants do not set aside germ cells early in development. In angiosperm species, reproduction occurs in the adult plant upon flowering. The multicellular male and female gametophytes differentiate from meiotic products within reproductive floral organs. Double fertilization is another remarkable feature of most angiosperm species. The zygote derived from fertilization of the egg cell by one of the sperm cells and the endosperm from fertilization of the central cell by the second sperm cell develop in a coordinated manner together and enclosed in the sporophytic maternal integuments, forming the seed. Understanding plant reproduction is biologically pertinent and agronomically and ecologically important. Here, we describe the known functions of histone lysine methylations in various steps of reproduction in the reference plant Arabidopsis thaliana. It is emerging that histone lysine methylation is key for understanding epigenetic regulation networks of genome function.
\end{abstract}

epigenetics, chromatin, histone methylation, SET domain, methyltransferase, Arabidopsis thaliana

Citation: Yao X Z, Shen W H. Crucial function of histone lysine methylation in plant reproduction. Chinese Sci Bull, 2011, 56: 3493-3499, doi: $10.1007 / \mathrm{s} 11434-011-4814-3$

In eukaryotes, DNA is packaged into a structure called chromatin. The fundamental unit of chromatin is nucleosome, which is composed of 146 base pairs of DNA wrapped around a histone octamer (two copies of each histone proteins $\mathrm{H} 2 \mathrm{~A}, \mathrm{H} 2 \mathrm{~B}, \mathrm{H} 3$, and $\mathrm{H} 4$ ). The structure properties of chromatin determine the DNA accessibility to various types of protein factors and RNA molecules and thus greatly influence the genome function. Epigenetics refer to heritable (mitotically and/or meiotically) changes in genome function that occur without a change in DNA sequence. Well-known epigenetic mechanisms include posttranslational covalent modifications (e.g. methylation and ubiquitylation) of histone tails, histone variant replacement, ATP-dependent chromatin remodelling and DNA methylation at cytosine residues.

Methylation of lysine residues on the tails of histones plays key roles in the regulation of gene expression and chromatin structure in eukaryotes. In Arabidopsis, histone lysine methylation occurs mainly at histone $\mathrm{H} 3$ lysines 4, 9,
27 and 36 (H3K4, H3K9, H3K27 and H3K36) [1]. In general, H3K4 and H3K36 methylation correlates with transcriptional activation whereas $\mathrm{H} 3 \mathrm{~K} 9$ and $\mathrm{H} 3 \mathrm{~K} 27$ methylation associates with gene silencing. In addition, each lysine can be mono-, di- or tri-methylated, and these different methylation states could have distinct physiological effects [2]. Histone methylations are carried out by histone methyltransferases that contain an evolutionarily conserved domain, the SET (named after SU(VAR) 3-9, E(Z) and TRITHORAX in Drosophila) domain. The reference plant Arabidopsis thaliana contains over 30 genes encoding SET-domain proteins [3], and so far only some of these genes have been functionally characterized [4,5].

The plant life cycle alternates between diploid sporophytic generation and a much reduced haploid gametophytic generation. In most of sexually reproducing plants including Arabidopsis, the gametophytic generation occurs late in development within sporophytic tissues of specialized floral organs. After pollination, the male and female gametes fuse

*Corresponding authors (email: xiaozhenyao@163.com; wen-hui.shen@ibmp-cnrs.unistra.fr) 
to produce diploid zygote and triploid endosperm. Histone methylations play pivotal roles throughout the plant life cycle. Here, we discuss crucial functions of histone lysine methylations in Arabidopsis reproduction, including flowering transition, gametophyte development, fertilization and seeds development.

\section{Histone lysine methylations in flowering transition}

Flowering, which represents the transition from the vegetative to reproductive development, is critical to reproductive success. The Arabidopsis flowering transition is controlled by four major regulatory pathways, namely photoperiod, vernalization, gibberellic acid signaling, and autonomous pathways [6]. FLOWERING LOCUS C (FLC), a MADS box transcription factor, is a major repressor of flowering and plays a central role in flowering-time control $[7,8]$. Several SET DOMAIN GROUP (SDG) proteins are involved in deposition of either 'active' or 'repressive' methylation marks at $F L C$ chromatin, and a fine-tuned chromatin remodeling at $F L C$ is crucial for flowering time control (Figure 1).

\subsection{H3K36 and H3K4 methylations in activation of FLC expression}

SDG8/ASHH2, also known as Early Flowering in Short days (EFS), is the first $S D G$ gene shown to be involved in activation of $F L C$ expression $[9,10]$. The $s d g 8$ mutants display an early-flowering phenotype, which is associated with reduced levels of FLC expression [9-11]. Ectopic expression of $F L C$ is sufficient to inhibit the early-flowering phenotype in $s d g 8$ mutant background [10]. Arabidopsis has 5 FLC paralogs, namely MADS AFFECTING FLOWERING1 (MAF1) to MAF5. While $M A F 2$ and $M A F 3$ expression is barely affected, expression of MAFl, MAF4 and $M A F 5$ is strongly reduced in the $s d g 8$ mutants [11]. Nevertheless, whether reduced $M A F$ gene expression contributes to the $s d g 8$ mutant phenotype remains unexamined. The SDG8 protein shows sequence homologies with the yeast H3K36-methyltransferase SET1/SET2. Consistently, a reduced level of H3K36me2 was observed in global histone extract as well as at the FLC and MAF loci in the $s d g 8 \mathrm{mu}-$ tants $[10,11]$. Interestingly, while the level of H3K36me3 was also reduced, H3K36me1 accumulated to higher levels in the $s d g 8$ mutants, indicating that SDG8 is responsible for deposition of H3K36me2 and H3K36me3 but not H3K36me1 [11]. This also establishes that the levels of H3K36me 2 and H3K36me3 but not H3K36me1 positively correlate with transcriptional activation of FLC and MAF genes. It will be obviously interesting to understand how the H3K36 methylation states are controlled and how the SDG8 protein is targeted to FLC and MAF loci.

The SDG26/ASHH1 protein is smaller and shares sequence homologies with only the C-terminal part of SDG8/ ASHH2, including the SET domain. Strikingly, the sdg26

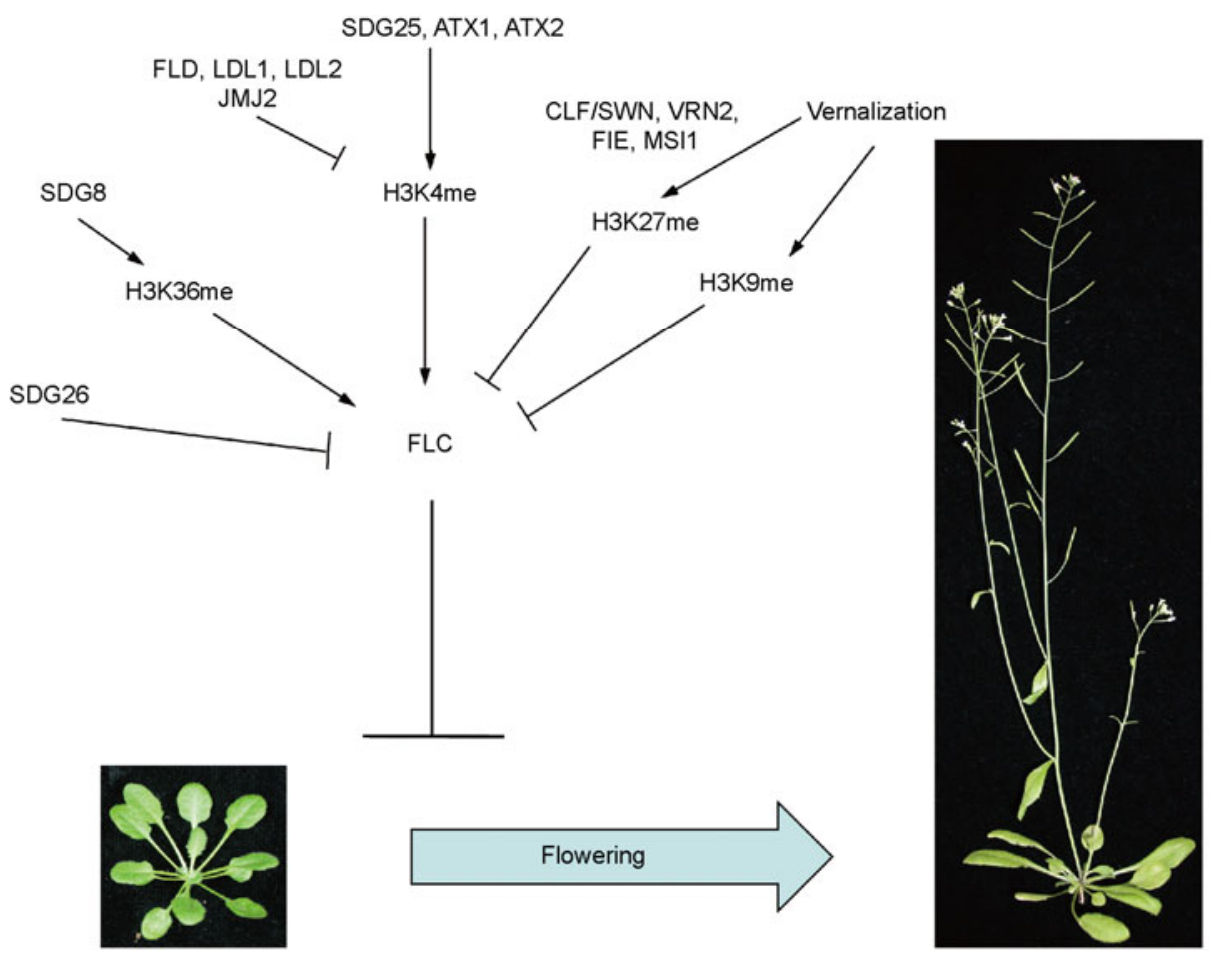

Figure 1 Histone methylations fine-tune $F L C$ expression in the control of transition to flowering. Solid lines with arrows indicate up-regulation or activation of gene expression, and solid lines with bars for down-regulation or repression of gene expression. 
mutants, opposite to the $s d g 8$ mutants, show a late-flowering phenotype and up-regulation of FLC, MAF4 and MAF5 [11]. Although the recombinant SDG26 protein could methylate oligonucleosomes in vitro, histone methylation levels were barely affected in the $s d g 26$ mutant plants. Molecular mechanisms involved in the SDG26-mediated repression of $F L C$ and $M A F$ genes remain obscure so far.

In addition to SDG8 and SDG26, three members of the Trithorax (TRX) group in Arabidopsis, ATX1/SDG27, ATX2/SDG30 and ARABIDOPSIS TRITHORAXRELATED7 (ATXR7)/SDG25, also contribute to flowering transition control. The atxl mutant shows a weak earlyflowering phenotype, which is enhanced by additional knockdown of ATX2 [12]. The ATX1 protein directly interacts with $F L C$ chromatin and is involved in the H3K4me3 deposition at the FLC locus [12]. FRIGIDA (FRI), a coiled-coil protein, acts to activate $F L C$ expression and inhibits flowering [13]. It has been reported that WDR5a, a homology of human WDR5 (one of the 4 core components of the MLL1 complex, which catalyzes H3K4 methylation), interacts with ATX1 in the yeast two-hybrid assay, and is required for FRI-mediated $\mathrm{H} 3 \mathrm{~K} 4 \mathrm{me} 3$ deposition at FLC chromatin [14]. The FRI-based complex also contains SDG8/EFS involved in H3K36me3 deposition at FLC chromatin $[15,16]$. Although ATX1 and ATX2 share high sequence homologies, ATX1 is involved in H3K4me3 whereas ATX2 in H3K4me2 deposition [17]. Nevertheless, whether ATX2 and ATX1 act sequentially on H3K4me2 and $\mathrm{H} 3 \mathrm{~K} 4 \mathrm{me} 3$ deposition at $F L C$ remains unexamined so far.

Compared to ATX1 and ATX2, SDG25/ATXR7 plays a more substantial role in flowering repression. The $s d g 25 / a t x r 7$ mutants show an obvious early-flower phenotype and reduced levels of FLC expression [18,19]. The recombinant SDG25/ATXR7 protein could methylate oligonucleosomes in vitro [18] and the SDG25/ATXR7-GFP protein was located around the transcript start site of $F L C$ [19]. Modest reduction of $\mathrm{H} 3 \mathrm{~K} 4 \mathrm{me} 2, \mathrm{H} 3 \mathrm{~K} 4 \mathrm{me} 3$ and H3K36me2 levels was observed at FLC in the $s d g 25 / a t x r 7$ mutant plants $[18,19]$. The atxl sdg25/atxr7 double mutant showed an enhanced early-flowering phenotype and more drastic reduction of $\mathrm{H} 3 \mathrm{~K} 4 \mathrm{me} 2$ and $\mathrm{H} 3 \mathrm{~K} 4 \mathrm{me} 3$ at $F L C$ [19]. Molecular interaction between ATX1 and SDG25/ATXR7 remains unclear.

\subsection{H3K27 methylation in repression of $F L C$ expression}

Polycomb Group (PcG) proteins were initially characterized in Drosophila melanogaster through their crucial function in silencing of homeotic (Hox) genes [20]. They exist in multi-protein complexes; the best characterized are known as polycomb repressive complex1 (PRC1) and PRC2. PRC2 is responsible for $\mathrm{H} 3 \mathrm{~K} 27 \mathrm{me} 3$ deposition and is composed of 4 core proteins, e.g. Enhancer of zeste $(\mathrm{E}(\mathrm{z})$, an H3K27- methyltransferase), Suppressor of zeste $12(\mathrm{Su}(\mathrm{z}) 12)$, Extra sex comb (Esc) and p55 [20]. H3K27me3 is recognized by the core component Pc of the PRC1 complex, and PRC1 further modifies chromatin by $\mathrm{H} 2 \mathrm{~A}$ monoubiquitylation [20]. The composition of PRC2 is conserved in Arabidopsis [21]. Homologs of all 4 core members of PRC2 have been identified in Arabidopsis: CURLY LEAF (CLF/SDG27), MEDEA (MEA/SDG1), and SWINGER (SWN/SDG30) are $\mathrm{E}(\mathrm{Z})$ homologs; FERTILIZATION INDEPENDENT SEED2 (FIS2), EMBRYONIC FLOWER2 (EMF2), and VERNALIZATION2 (VRN2) are Su(z)12 homologs; MULTICOPY SUPPRESSOR OF IRA (MSI) 1-5 are p55 homologs; and FERTILIZATION-INDEPENDENT ENDOSPERM (FIE) is the only one homolog of Esc [21]. More recently, PRC1like complexes were also characterized in Arabidopsis and shown to play essential roles in plant development [22-24].

Prolonged exposure in cold (vernalization) during winter is memorized for blooming in spring in many plant species, including some agronomically important cereals. In Arabidopsis, vernalization promotes flowering largely by repression of FLC expression [25]. Vernalization increases $\mathrm{H} 3 \mathrm{~K} 9 \mathrm{me} 2, \mathrm{H} 3 \mathrm{~K} 27 \mathrm{me} 2$ and $\mathrm{H} 3 \mathrm{~K} 27 \mathrm{me} 3$ accumulation at FLC locus [26]. PcG proteins VRN2, FIE, CLF/SDG27, SWN/SDG30, MSI1 and the plant homeodomain proteins VERNALIZATION INSENSITIVE 3 (VIN3) and VIN3like1 (VIL1/VRN5) form large protein complexes involved in the H3K27me3 deposition on FLC chromatin during vernalization [27]. H3K27me3 spreads across the $F L C$ locus in a DNA replication dependent manner [28]. The PRC1like component, TERMINAL FLOWER2 (TFL2)/LIKE HETEROCHROMATIN PROTEIN1 (LHP1), associates with chromatin regions enriched of $\mathrm{H} 3 \mathrm{~K} 27 \mathrm{me} 3$ and is involved in the maintenance of FLC repression [29]. The flowering phenotype caused by knockdown of $P c G$ genes is complex. Opposite to a late-flowering phenotype predictable by release of FLC repression, the $c l f$, emf 2 and $l h p l$ mutants display a strong early-flowering phenotype. This is now understood because that PRC2 and LHP1 play key roles in repression of FLOWERING LOCUS T (FT), a major flowering activator acting at downstream of $F L C$ in the regulatory pathway [30].

\subsection{Fine-tuning histone lysine methylations in regulation of $F L C$ expression}

Distinct from single mutants, the atxl clf double mutant showed a phenotype indistinguishable from wide-type plants [31]. It was shown that ATX1 and CLF proteins interact physically with each other in the yeast two-hybrid assay [31]. This study unravels antagonistic roles of ATX1 and CLF. Nevertheless, much progress needs to be made to understand how different histone methylations mark the FLC chromatin spatially and timely in the flowering time control.

Histone methylations written by SDG histone methyl- 
transferases can be erased by histone demethylases. FCA, an RNA-binding protein, plays roles in the autonomous pathway for flowering time control. FLOWERING LOCUS D (FLD), a plant homolog of Lysine-Specific Demethylase1 (LSD1, a H3K4-demethylase), is required for FCA to down-regulate $F L C$ expression and promote flowering [32]. The other two homologues of LSD1, LSD1-LIKE1 (LDL1) and LSD1-LIKE2 (LDL2), also play partially redundant roles with FLD in the regulation of floral transition [33]. JmjC-domain proteins are known to have histone demethylase activity. So far, three proteins that belong to Jumonji class have been shown to control the flowering transition. RELATIVE OF EARLY FLOWERING6 (REF6)/AtJmj2 acts as an FLC repressor, and the ref6 mutant shows late flowering phenotype under both long-day and short-day growth conditions [34]. AtJmj4/14 and EARLY FLOWERING6 (ELF6)/AtJmj1, acting as H3K4 demethylases, repress $F T$ expression and prevent precocious flowering in Arabidopsis [35,36]. It was reported that ELF6/ AtJmj1 and AtJmj4/14 bind FT chromatin and that H3K4 methylations are increased in the elf6 and jmj4 and more pronouncedly in the double elf6 jmj4 mutants [35]. Increase of $\mathrm{H} 3 \mathrm{~K} 4 \mathrm{me} 2 / \mathrm{me} 3$ levels was also observed at FLC chromatin in the ref6 and fld mutants [15].

Small interfering RNA (siRNA) could regulate transcription via histone methylation. In the Arabidopsis Ler accession, FLC contains a transposable element inserted in an intron of the gene, and siRNAs were shown to be involved in targeting $F L C$ for repressive $\mathrm{H} 3 \mathrm{~K} 9$ methylation [37]. In the Arabidopsis Columbia (Col) accession, two small RNAs corresponding to the reverse strand were identified and shown to be involved in $\mathrm{H} 3 \mathrm{~K} 9$ methylation at $F L C$ in a DICER-LIKE2 (DCL2)/DCL3/DCL4-dependent manner [38]. Interestingly, mutations for a single $D C L$ do not trigger a strong flowering phenotype, while double $d c l 1 ~ d c l 3$ mutants are late flowering due to an increase of FLC expression [39]. The identity of SDG(s) involved in $\mathrm{H} 3 \mathrm{~K} 9$ methylation at FLC locus remains currently unknown. Recently, a long intronic noncoding RNA, named COLD ASSISTED INTRONIC NONCODING RNA (COLDAIR), was shown to be required for the vernalization-mediated epigenetic repression of FLC [40]. COLDAIR physically associates with CLF, suggesting that COLDAIR establishes stable repressive chromatin at $F L C$ through recruiting PRC2 in H3K27me3 deposition [40].

\section{Function of histone lysine methylations in gametophyte development}

Female gametophytes or megagametophytes develop in ovules within the gynoecium of the Arabidopsis flower. A single megaspore mother cell (megasporocyte) differentiates from the subepidermal cell layer at the tip of each ovule primordium and undergoes meiosis to produce a tetrad of four haploid spores. Three of the spores degenerate, and one proceeds through 3 sequential rounds of mitotic division, forming the female gametophyte, the embryo sac, which at maturation consists of 7 cells with four cell types ( 3 antipodal cells, 2 synergid cells, 1 egg cell and 1 two-haploid-fused diploid central cell). The male gametophytes or microgametophytes develop within anthers of the Arabidopsis flower. Microsporocytes differentiate from the primary sporogenous tissue surrounded by the tapetum and undergo meiosis to form a tetrad of four haploid microspores. The nucleus of each microspore follows one cycle of nuclear division, forming a generative cell and a vegetative cell. The generative cell undergoes one more round of mitosis to produce two sperm cells. At maturation the male gametophyte, the pollen grain, is thus composed of a three-celled male germ unit.

Besides controlling the flowering time, SDG8/ASHH2 is also involved in floral organ as well as male and female gametophyte development [11,41]. It was reported that about $80 \%$ of $s d g 8 / a s h h 2$ mutant mature ovules lack an embryo sac [41]. As to the male gametophyte development, the tapetum development was affected and the number of functional pollen per anther is drastically reduced in $s d g 8 / a s h h 2$. Furthermore, the transmission efficiency of sdg8/ashh2 was reduced through both male and female gametes. Reduced levels of H3K36me3 were observed at the floral organ identity regulatory gene $A P 1$, the meiosis essential gene AtDMCI and the pollen development regulatory gene $M Y B 99$, suggesting that SDG8/ASHH2-mediated H3K36me3 deposition is important for activation of these genes, which is required during various processes of reproduction [41].

More recent characterization of the SDG2/ATXR3 gene revealed that $\mathrm{H} 3 \mathrm{~K} 4$ methylation is crucial for plant reproduction $[42,43]$. Compared to the partial sterile phenotype of the $s d g 8 / a s h h 2$ mutants, the $s d g 2$ mutants are completely sterile. SDG2 is a methyltransferase responsible for the global genome-wide $\mathrm{H} 3 \mathrm{~K} 4 \mathrm{me} 3$ deposition. It is involved in multiple developmental processes, remarkably important in initiation processes of female and male gametophyte development [42]. Although both $s d g 2$ and $s d g 8 / a s h h 2$ mutants show floral organ and gametophyte defects, they also display obvious differences. Future analysis by the use of developmental marker genes might be helpful to precise $S D G 2$ and SDG8/ASHH2 roles during gametogenesis. Transcriptome analyses of young flower buds have identified a few hundred genes differentially regulated by more than 2 -fold in either $s d g 2-1$ or $s d g 8 / a s h h 2$ mutant $[41,42]$. Only less than $20 \%$ of differentially expressed genes are in common between the two mutants and these are primarily involved in metabolism and transport, indicating that SDG2 and SDG8/ ASHH2 may regulate flower development through common and distinct gene networks. Among the SDG2-target genes, the MADS-like transcription factor gene SPOROCYTELESS/ NOZZLE (SPL/NZZ) is of particular interests [42]. This gene acts upstream of many stamen-expressed and ovule- 
expressed genes and is required for initiation of both microsporogenesis and megasporogenesis [44,45]. The only previous known gene that acts upstream of $S P L / N Z Z$ is the reproductive organ identity gene AGAMOUS $(A G)$, which activates $S P L / N Z Z$ expression through binding to the 3 '-region of the SPL/NZZ gene [46]. The SPL/NZZ locus contains bivalent chromatin domains consisting of the transcriptional active mark $\mathrm{H} 3 \mathrm{~K} 4 \mathrm{me} 3$ and the transcriptional repressive mark $\mathrm{H} 3 \mathrm{~K} 27 \mathrm{me} 3$ [42]. SDG2 is required for marking $\mathrm{H} 3 \mathrm{~K} 4 \mathrm{me} 3$ for active transcription of $S P L / N Z Z$. However, PcG genes involved in marking H3K27me3 at SPL/NZZ remains currently unknown. Study of SDG2mediated activation and PcG-mediated repression of SPL/NZZ might provide a key step for understanding gene regulatory networks in gametophyte development.

\section{Function of hisone lysine methylations in seed development}

Double fertilization is a specific event during plant reproduction. After pollination, the pollen tube delivers two sperm cells into embryo sac. One of the sperm cell fuses with egg cell, leading to development of a diploid embryo. Fusion of the other sperm cell with central cell generates the triploid endosperm, a nutritive tissue that supports embryogenesis. It has been reported that histone methylations play important roles in fertilization and seeds development.

Mutants fie, fis 2 and mea/sdg1 show a similar phenotype that is autonomous endosperm development without fertilization [47-49]. The mea embryos display excess cell proliferation and enlarge radial symmetrically from later globular stage. $M E A$ also controls the endosperm development through suppression of proliferation of the central cell before fertilization. When fertilization is prevented by remove the anther, the mea endosperm proliferates without embryo development [47,49]. The mea seeds turn white and collapsed during seed desiccation, and such kind of abnormality is independent of the paternal contribution and gene dosage. $M E A$ is one of the best-characterized imprinted genes in plants. The maternal allele of MEA is hypomethylated and is actively expressed in the endosperm. A PRC2 complex, composed of FIE, FIS2, MSI1 and maternalexpressed MEA, is proposed to silence through H3K27methylation the paternal MEA allele in endosperm cells [50]. This same complex is also likely responsible for suppression of central cell proliferation before fertilization. Similar to mea, fie and msil shows endosperm proliferation independent from fertilization. In msil mutant, the development of the embryos surrounded by diploid endosperm will arrested at different stages after pollination [51]. The swn/ sdg30 mutant alone does not show obvious defects; it can, however, enhance the mea mutant in producing fertilization independent seeds [52], indicating that additional PRC2 complexes may be involved in seed development control.
One of the targets of the MEA-based PRC2 complex is the MADS-box gene PHERES1 (PHE1) [53]. In contrast to maternally expressed $M E A, P H E 1$ is preferentially expressed in endosperm from the paternal allele. The vegetative and reproductive development of phel mutants was indistinguishable from that of wild-type plants. Interestingly, the repression of the maternal PHEI allele is disrupted in mea mutant seeds with mutated maternal MEA allele [54]. MEA and FIE directly bind with the PHE1 promoter and repress the maternal PHE1 allele through H3K27me3 deposition [53,54].

By monitoring in situ in the ovule the dynamics of chromatin states in the female gametes, Pillot et al. [55] showed that H3K9me2 and LHP1 are more abundantly present in the egg cell than in the central cell, which pre-establishes the differentiation that the embryo is relatively quiescent, while the endosperm is transcriptionally active. KRYPTONITE (KYP)/SDG33 is the methyltransferase primarily involved in $\mathrm{H} 3 \mathrm{~K} 9 \mathrm{me} 2$ deposition in the embryo [55]. At $8 \mathrm{~d}$ after pollination, the entire endosperm genome is demethylated, which is in sharp contrast with the methylated embryo genome $[56,57]$. It thus appears that $\mathrm{H} 3 \mathrm{~K} 9 \mathrm{me} 2$ associates with DNA methylation, contributing to the transcriptionally repressive heterochromatic state in the embryo, whereas hypomethylation of DNA and $\mathrm{H} 3 \mathrm{~K} 9$ are virtually linked to the permissive euchromatic state in the endosperm.

\section{Concluding remarks}

Studies over the last few years have shown that SDGsmediated histone methylations play crucial roles in plant reproduction. The key flowering time regulator FLC is emerging as one of the most studied loci in epigenetic research. Because of its bivalent chromatin nature and its critical function in initiation of both microsporogenesis and megasporogenesis, $S P L / N Z Z$ may become a target of choice in future research. It is predictable that more factors will be identified as being involved in writing, reading and erasing histone methylations in the regulation of FLC and SPL/NZZ transcription. Still very little is known about how different factors involved in histone methylations are recruited to their chromatin targets. The interactionships between different factors in many cases remain elusive or in some are totally unknown. We also need to dissect dynamics of histone methylations in temporal and tissue or cell-type specific manner. It will be challenging to understand how a histone methylation state is established and inherited during mitosis and/or meiosis, and how resetting of chromatin state occurs during reproduction.

1 Johnson L, Mollah S, Garcia B A, et al. Mass spectrometry analysis of Arabidopsis histone H3 reveals distinct combinations of posttranslational modifications. Nucleic Acids Res, 2004, 32: 6511-6518

2 Martin C, Zhang Y. The diverse functions of histone lysine methylation. Nat Rev Mol Cell Biol, 2005, 6: 838-849 
3 Baumbusch L O, Thorstensen T, Krauss V, et al. The Arabidopsis thaliana genome contains at least 29 active genes encoding SET domain proteins that can be assigned to four evolutionarily conserved classes. Nucleic Acids Res, 2001, 29: 4319-4333

4 Liu C, Lu F, Cui X, et al. Histone methylation in higher plants. Annu Rev Plant Biol, 2010, 61: 395-420

$5 \mathrm{Yu} \mathrm{Y,} \mathrm{Pu} \mathrm{Z,} \mathrm{Shen} \mathrm{W} \mathrm{H,} \mathrm{et} \mathrm{al.} \mathrm{An} \mathrm{update} \mathrm{on} \mathrm{histone} \mathrm{lysine} \mathrm{methyla-}$ tion in plants. Prog Nat Sci, 2009, 19: 407-413

6 Baurle I, Dean C. The timing of developmental transitions in plants. Cell, 2006, 125: 655-664

7 Michaels S D, Amasino R M. FLOWERING LOCUS C encodes a novel MADS domain protein that acts as a repressor of flowering. Plant Cell, 1999, 11: 949-956

8 Sheldon C C, Burn J E, Perez P P, et al. The FLF MADS box gene: A repressor of flowering in Arabidopsis regulated by vernalization and methylation. Plant Cell, 1999, 11: 445-458

9 Kim S Y, He Y, Jacob Y, et al. Establishment of the vernalization-responsive, winter-annual habit in Arabidopsis requires a putative histone H3 methyl transferase. Plant Cell, 2005, 17: 3301-3310

10 Zhao Z, Yu Y, Meyer D, et al. Prevention of early flowering by expression of FLOWERING LOCUS C requires methylation of histone H3 K36. Nat Cell Biol, 2005, 7: 1256-1260

11 Xu L, Zhao Z, Dong A, et al. Di- and tri- but not monomethylation on histone H3 lysine 36 marks active transcription of genes involved in flowering time regulation and other processes in Arabidopsis thaliana. Mol Cell Biol, 2008, 28: 1348-1360

12 Pien S, Fleury D, Mylne J S, et al. ARABIDOPSIS TRITHORAXI dynamically regulates FLOWERING LOCUS C activation via histone 3 lysine 4 trimethylation. Plant Cell, 2008, 20: 580-588

13 Johanson U, West J, Lister C, et al. Molecular analysis of FRIGIDA, a major determinant of natural variation in Arabidopsis flowering time. Science, 2000, 290: 344-347

14 Jiang D, Gu X, He Y. Establishment of the winter-annual growth habit via FRIGIDA-mediated histone methylation at FLOWERING LOCUS C in Arabidopsis. Plant Cell, 2009, 21: 1733-1746

15 Ko J H, Mitina I, Tamada Y, et al. Growth habit determination by the balance of histone methylation activities in Arabidopsis. EMBO J, 2010, 29: 3208-3215

16 Choi K, Kim J, Hwang H J, et al. The FRIGIDA complex activates transcription of FLC, a strong flowering repressor in Arabidopsis, by recruiting chromatin modification factors. Plant Cell, 2011, 23: 289-303

17 Saleh A, Alvarez-Venegas R, Yilmaz M, et al. The highly similar Arabidopsis homologs of trithorax ATX1 and ATX2 encode proteins with divergent biochemical functions. Plant Cell, 2008, 20: 568-579

18 Berr A, Xu L, Gao J, et al. SET DOMAIN GROUP25 encodes a histone methyltransferase and is involved in FLOWERING LOCUS C activation and repression of flowering. Plant Physiol, 2009, 151: 1476-1485

19 Tamada Y, Yun J Y, Woo S C, et al. ARABIDOPSIS TRITHORAXRELATED7 is required for methylation of lysine 4 of histone $\mathrm{H} 3$ and for transcriptional activation of FLOWERING LOCUS C. Plant Cell, 2009, 21: 3257-3269

20 Schuettengruber B, Chourrout D, Vervoort M, et al. Genome regulation by polycomb and trithorax proteins. Cell, 2007, 128: 735-745

21 Kohler C, Villar C B. Programming of gene expression by Polycomb group proteins. Trends Cell Biol, 2008, 18: 236-243

$22 \mathrm{Xu} \mathrm{L}$, Shen W H. Polycomb silencing of KNOX genes confines shoot stem cell niches in Arabidopsis. Curr Biol, 2008, 18: 1966-1971

23 Bratzel F, Lopez-Torrejon G, Koch M, et al. Keeping cell identity in Arabidopsis requires PRC1 RING-finger homologs that catalyze H2A monoubiquitination. Curr Biol, 2010, 20: 1853-1859

24 Chen D, Molitor A, Liu C, et al. The Arabidopsis PRC1-like ringfinger proteins are necessary for repression of embryonic traits during vegetative growth. Cell Res, 2010, 20: 1332-1344

25 Sung S, Amasino R M. Remembering winter: Toward a molecular understanding of vernalization. Annu Rev Plant Biol, 2005, 56: 491508

26 Bastow R, Mylne J S, Lister C, et al. Vernalization requires epige- netic silencing of FLC by histone methylation. Nature, 2004, 427: 164-167

27 Wood C C, Robertson M, Tanner G, et al. The Arabidopsis thaliana vernalization response requires a polycomb-like protein complex that also includes VERNALIZATION INSENSITIVE 3. Proc Natl Acad Sci USA, 2006, 103: 14631-14636

28 Finnegan E J, Dennis E S. Vernalization-induced trimethylation of histone $\mathrm{H} 3$ lysine 27 at FLC is not maintained in mitotically quiescent cells. Curr Biol, 2007, 17: 1978-1983

29 Mylne J S, Barrett L, Tessadori F, et al. LHP1, the Arabidopsis homologue of HETEROCHROMATIN PROTEIN1, is required for epigenetic silencing of FLC. Proc Natl Acad Sci USA, 2006, 103: 5012-5017

30 Jiang D, Wang Y, He Y. Repression of FLOWERING LOCUS C and FLOWERING LOCUS $T$ by the Arabidopsis Polycomb repressive complex 2 components. PLoS ONE, 2008, 3: e3404

31 Alvarez-Venegas R, Avramova Z. Methylation patterns of histone H3 Lys 4, Lys 9 and Lys 27 in transcriptionally active and inactive Arabidopsis genes and in atxl mutants. Nucleic Acids Res, 2005, 33: 5199-5207

32 Liu F, Quesada V, Crevillen P, et al. The Arabidopsis RNA-binding protein FCA requires a lysine-specific demethylase 1 homolog to downregulate FLC. Mol Cell, 2007, 28: 398-407

33 Jiang D, Yang W, He Y, et al. Arabidopsis relatives of the human lysine-specific Demethylase1 repress the expression of $F W A$ and FLOWERING LOCUS C and thus promote the floral transition. Plant Cell, 2007, 19: 2975-2987

34 Noh B, Lee S H, Kim H J, et al. Divergent roles of a pair of homologous jumonji/zinc-finger-class transcription factor proteins in the regulation of Arabidopsis flowering time. Plant Cell, 2004, 16: 2601-2613

35 Jeong $\mathrm{J} \mathrm{H}$, Song $\mathrm{H}$ R, Ko J H, et al. Repression of FLOWERING LOCUS $T$ chromatin by functionally redundant histone $\mathrm{H} 3$ lysine 4 demethylases in Arabidopsis. PLoS ONE, 2009, 4: e8033

36 Lu F, Cui X, Zhang S, et al. JMJ14 is an H3K4 demethylase regulating flowering time in Arabidopsis. Cell Res, 2010, 20: 387-390

37 Liu J, He Y, Amasino R, et al. siRNAs targeting an intronic transposon in the regulation of natural flowering behaviour in Arabidopsis thaliana. Genes Dev, 2004, 18: 2873-2878

38 Swiezewski S, Crevillen P, Liu F, et al. Small RNA-mediated chromatin silencing directed to the $3^{\prime}$ region of the Arabidopsis gene encoding the developmental regulator, FLC. Proc Natl Acad Sci USA, 2007, 104: 3633-3638

39 Schmitz R J, Hong L, Fitzpatrick K E, et al. DICER-LIKE 1 and DICER-LIKE 3 redundantly act to promote flowering via repression of FLOWERING LOCUS C in Arabidopsis thaliana. Genetics, 2007, 176: 1359-1362

40 Heo J B, Sung S. Vernalization-mediated epigenetic silencing by a long intronic noncoding RNA. Science, 2011, 331: 76-79

41 Grini P E, Thorstensen T, Alm V, et al. The ASH1 HOMOLOG 2 (ASHH2) histone $\mathrm{H} 3$ methyltransferase is required for ovule and anther development in Arabidopsis. PLoS ONE, 2009, 4: e7817

42 Berr A, McCallum E J, Menard R, et al. Arabidopsis SET DOMAIN GROUP2 is required for $\mathrm{H} 3 \mathrm{~K} 4$ trimethylation and is crucial for both sporophyte and gametophyte development. Plant Cell, 2010, 22: 3232-3248

43 Guo L, Yu Y, Law J A, et al. SET DOMAIN GROUP2 is the major histone H3 lysine 4 trimethyltransferase in Arabidopsis. Proc Natl Acad Sci USA, 2010, 107: 18557-18562

44 Schiefthaler U, Balasubramanian S, Sieber P, et al. Molecular analysis of NOZZLE, a gene involved in pattern formation and early sporogenesis during sex organ development in Arabidopsis thaliana. Proc Natl Acad Sci USA, 1999, 96: 11664-11669

45 Yang W C, Ye D, Xu J, et al. The SPOROCYTELESS gene of Arabidopsis is required for initiation of sporogenesis and encodes a novel nuclear protein. Genes Dev, 1999, 13: 2108-2117

46 Ito $\mathrm{T}$, Wellmer $\mathrm{F}, \mathrm{Yu} \mathrm{H}$, et al. The homeotic protein AGAMOUS controls microsporogenesis by regulation of SPOROCYTELESS. Nature, 2004, 430: 356-360 
47 Grossniklaus U, Vielle-Calzada J P, Hoeppner M A, et al. Maternal control of embryogenesis by $M E D E A$, a polycomb group gene in $A r$ abidopsis. Science, 1998, 280: 446-450

48 Luo M, Bilodeau P, Koltunow A, et al. Genes controlling fertilization-independent seed development in Arabidopsis thaliana. Proc Natl Acad Sci USA, 1999, 96: 296-301

49 Kiyosue T, Ohad N, Yadegari R, et al. Control of fertilizationindependent endosperm development by the MEDEA polycomb gene in Arabidopsis. Proc Natl Acad Sci USA, 1999, 96: 4186-4191

50 Huh J H, Bauer M J, Hsieh T F, et al. Cellular programming of plant gene imprinting. Cell, 2008, 132: 735-744

51 Kohler C, Hennig L, Bouveret R, et al. Arabidopsis MSI1 is a component of the MEA/FIE Polycomb group complex and required for seed development. EMBO J, 2003, 22: 4804-4814

52 Wang D, Tyson M D, Jackson S S, et al. Partially redundant functions of two SET-domain polycomb-group proteins in controlling ini- tiation of seed development in Arabidopsis. Proc Natl Acad Sci USA, 2006, 103: 13244-13249

53 Kohler C, Hennig L, Spillane C, et al. The Polycomb-group protein MEDEA regulates seed development by controlling expression of the MADS-box gene PHERES1. Genes Dev, 2003, 17: 1540-1553

54 Kohler C, Page D R, Gagliardini V, et al. The Arabidopsis thaliana MEDEA Polycomb group protein controls expression of PHERES1 by parental imprinting. Nat Genet, 2005, 37: 28-30

55 Pillot M, Baroux C, Vazquez M A, et al. Embryo and endosperm inherit distinct chromatin and transcriptional states from the female gametes in Arabidopsis. Plant Cell, 2010, 22: 307-322

56 Gehring M, Bubb K L, Henikoff S. Extensive demethylation of repetitive elements during seed development underlies gene imprinting. Science, 2009, 12: 1447-1451

57 Hsieh T F, Ibarra C A, Silva P, et al. Genome-wide demethylation of Arabidopsis endosperm. Science, 2009, 324: 1451-1454

Open Access This article is distributed under the terms of the Creative Commons Attribution License which permits any use, distribution, and reproduction in any medium, provided the original author(s) and source are credited. 